\title{
Comportamiento del tiempo de duración, la frecuencia de los cortocircuitos y la conductividad eléctrica durante el reencendido del arco en la soldadura SMAW (AC) con electrodos E6013
}

\author{
(Behavior of short-circuit frequency and duration time and electrical conductivity on arc turn- on during SMAW (AC) with \\ E6013 electrodes)
}

\author{
Alejandro García Rodríguez¹, Carlos René Gómez Pérez¹, Oscar Miguel Rivera Borroto², Jorge Víctor Miguel Oria ${ }^{1}$ \\ ${ }^{1}$ Centro de Investigaciones de Soldadura. Universidad Central "Marta Abreu” de Las Villas. \\ ${ }^{2}$ Centro de Estudios Informáticos. Universidad Central de Las Villas. \\ alejo2@uclv.edu.cu, crene@uclv.edu.cu
}

\begin{abstract}
Resumen
El presente trabajo tiene como objetivo la evaluación del comportamiento del tiempo de duración, la frecuencia de los cortocircuitos y la conductividad durante el reencendido del arco en el proceso de soldadura SMAW (Shielded Metal Arc Welding), con corriente alterna y electrodos E6013. El análisis estadístico no-paramétrico garantiza un procesamiento robusto de los datos, atenuando la influencia de valores atípicos y errores derivados del empleo de aproximaciones a distribuciones continuas conocidas. La mediana y la mediana de la desviación absoluta (MAD), respecto a la mediana de los datos, constituyen los estimadores de localización y dispersión utilizados, respectivamente. El electrodo, en el régimen de $160 \mathrm{~A}$, presenta una mayor estabilidad, en el aporte metálico, dada por el menor valor del MAD promedio del período de cortocircuito $(39,36 \mathrm{~ms})$ y de la duración del cortocircuito (1,43 ms), reafirmada con la presencia de una mayor conductividad eléctrica durante el reencendido $\left(1766,17 \times 10^{-3} \mathrm{~S} \cdot \mathrm{s}^{-1}\right)$.
\end{abstract}

Palabras clave: SMAW, parámetros eléctricos, análisis estadístico, procesamiento robusto, desempeño, E6013

\begin{abstract}
The objective of this work is the valuation of the behavior of short-circuits frequency and duration time and electrical conductivity on arc reigniting in SMAW (Shielded Metal Arc Welding) process with alternate current and E6013 electrodes. The non parametric statistic analysis realize a robust data processing, minimizing the outliers influence and mistakes derivates about employ of approximations to well know continues distributions. The median and the median absolute deviation (MAD) respect to median of the data are the localization and dispersion estimators used, respectively. The electrode at 160 A present a better stability on metal transference supported on the most little value of MAD for the period of transference (39,36 ms), and the MAD of the short-circuit duration (1,43 ms), according with the presence of a major electric conductivity during the arc reigniting $\left(1766,17 x 10^{-3} S^{\cdot-1}\right)$.
\end{abstract}

Key-words: SMAW, electric parameters, statistical analysis, robust processing, behavior, E6013

\section{Introducción}

Algunos autores [1-8] han publicado sus experiencias en la caracterización operativa de electrodos revestidos en régimen de transferencia metálica por cortocircuito, mediante el empleo de la digitalización del voltaje y la corriente del arco.

El comportamiento de los parámetros eléctricos ha sido descrito esencialmente en el trabajo de Ponomarev [8], determinándose las diferencias fundamentales para los distintos tipos de revestimientos empleados para electrodos revestidos. Especialmente los electrodos rutílicos han sido objeto de estudio en países latinoamericanos [7] para la obtención de información sobre la operatividad de este tipo de electrodo de amplio uso a nivel mundial por su excelente comportamiento eléctrico.

Sin embargo, el procesamiento estadístico de los parámetros

(Recebido em 07/07/2008; Texto Final em 10/12/2008). no ha sido expuesto de manera explícita cuando se utilizan dispositivos de alimentación automática forzada [1, 3, 4, 7]. En el caso de nuestra investigación se utiliza un sistema de alimentación por gravedad que no introduce modificaciones en la respuesta propia del electrodo. La evaluación de la respuesta del electrodo bajo este sistema de autoalimentación impone el análisis de las distribuciones de los parámetros para determinar el procedimiento estadístico adecuado.

En la composición química del revestimiento del electrodo rutílico E6013, el óxido de titanio proporciona, mediante el titanio, un elemento de bajo potencial de ionización que garantiza un adecuado proceso encendido y reencendido del arco eléctrico, por lo que es reconocido como un electrodo de alta estabilidad eléctrica, muy útil en el trabajo con corriente alterna [2, 9], lo cual lo hace apropiado para el estudio de la influencia de los materiales del revestimiento en la estabilidad del arco eléctrico en la soldadura. Esencialmente el régimen de transferencia metálica por cortocircuito ocurre cuando se suelda con electrodos revestidos en la mayoría de las aplicaciones, alcanzándose 
las mayores tasas de deposición horaria, y variaciones en los parámetros definidos en las metodologías [1, 8], tales como: duración del cortocircuito, frecuencia de cortocircuito y conductividad eléctrica durante el reencendido del arco. El efecto producido por los diferentes gases componentes de la atmósfera que se forman por la descomposición térmica de los materiales del recubrimiento durante la soldadura, se refleja en las señales de voltaje y corriente del arco, de cuyo procesamiento se extraen los parámetros con determinadas distribuciones probabilísticas, que es necesario analizar y describir utilizando las medidas de tendencia central y dispersión más adecuadas [10].

\section{Materiales y métodos}

En la soldadura con electrodo consumible uno de los principios básicos para mantener constante la longitud del arco radica en garantizar que la velocidad de alimentación del electrodo coincida con la velocidad de fusión del mismo [10, 11]. Con este fin se utiliza un dispositivo de alimentación por gravedad $[6,10,12-15]$, que garantiza que las variaciones de la estabilidad del arco dependan exclusivamente de las características propias del electrodo revestido. Además, se utiliza una instalación compuesta por un transformador de soldadura [12], un circuito acondicionador de señales de voltaje y corriente, provenientes de la fuente de potencia en un rango de $-5 \mathrm{~V}$ a $+5 \mathrm{~V}$, acoplado en su salida a una computadora, donde las señales analógicas son digitalizadas mediante una tarjeta de adquisición de datos PCI-1710, con una frecuencia de muestreo de hasta $100 \mathrm{kHz}$ y un convertidor análogo digital de 12 bits [16], programada para obtener 5000 muestras por segundo en los canales de voltaje y corriente de soldadura durante el tiempo que dura el experimento.

Desde el punto de vista operativo, los electrodos rutílicos son conocidos como los mejores comparados con los celulósicos y los básicos, siendo ampliamente utilizados a nivel mundial [7].

\subsection{Proceder experimental}

Se utilizan electrodos de la clasificación E6013 [9] (Ø 4 $\mathrm{mm}$ ) contenidos en el mismo paquete sellado, certificados para su uso en corriente directa y corriente alterna, en un rango de 120A a 160A. Para variar el régimen de trabajo se opta por una planificación experimental correspondiente a un diseño factorial (Tabla 1) de 3 niveles para el factor independiente "Corriente", con dos réplicas independientes en cada nivel, realizándose posteriormente un análisis estadístico entre y dentro de grupos, según la terminología usada en Diseños de Experimentos tipo One-way analysis of Variante (ANOVA) [17]. Los 9 electrodos se escogen mediante un muestreo aleatorio, sin reposición, sometiéndose todos a un procedimiento de identificación, medida, pesaje e inspección visual, que garantiza que los mismos no presenten defectos, tales como grietas, roturas, excentricidad del revestimiento, así como un peso y dimensiones diferentes a los del electrodo promedio definido por el fabricante.
Tabla 1. Valores de corriente a los que se someten los electrodos en cada uno de los tres ensayos.

\begin{tabular}{c|c|c|c}
\hline Experimento & 1 & 2 & 3 \\
\hline Corriente de Soldadura (A) & 125 & 140 & 160 \\
\hline
\end{tabular}

La fuente de corriente alterna impone reiteradas inversiones de polaridad en el arco a un ritmo de 120 veces por segundo, convirtiéndose el electrodo en cátodo y la placa metálica en ánodo y viceversa, imponiéndose diferentes condiciones para la transferencia de calor que afectan la estabilidad térmica del arco [11]. Este efecto hace que se encienda y apague el arco eléctrico en sucesivas oportunidades, siendo idóneo para la evaluación del comportamiento eléctrico del consumible en condiciones de inestabilidad reiteradas.

Se realizaron soldaduras con corriente alterna sobre placas de 200x50x10 mm de acero al carbono CT-3, según las normas Rusas y Ucranianas “Gostandar”. El dispositivo de alimentación y las placas a soldar fueron sucesivamente fijados mediante tornillos a la mesa de trabajo obteniéndose nueve cordones de soldadura en posición plana, tres en cada régimen (Tabla1). La constancia en el coeficiente de forma del cordón y la situación de los frentes de solidificación indican una mayor estabilidad en el régimen de $160 \mathrm{~A}$ [12].

La instalación experimental consta de un transformador de soldadura AIRCO 220V AC, una mesa de trabajo, un circuito acondicionador de señales y una tarjeta de adquisición de datos acoplada a una computadora. La corriente, sensada mediante un SHUNT, y el voltaje de soldadura son acondicionados y acoplados mediante un sistema de adquisición de datos Advantech PCLD8710 de hasta $100 \mathrm{kHz}$, un convertidor A/D de 12 bits, 16 canales de entrada digital, 16 canales de salida digital, contador y temporizador programable y 2 canales de salida analógica, 16 canales de entrada simple u 8 entradas diferenciales o combinaciones; una frecuencia de muestreo de 100 mil muestras por segundo; tiempo de conversión 8 microsegundos; tensión máxima de entrada, $\pm 15 \mathrm{~V}$; impedancia de entrada, 1 millón de Ohms por cada 5 pico Faradios.

Las señales de voltaje y corriente fueron digitalizadas con una frecuencia de 5000 muestras por segundo sin distorsión de frecuencia ni fase y con un error inferior al $2 \%$ en la medición de amplitud.

Se empleó un dispositivo de alimentación por gravedad, que elimina la influencia del sistema de alimentación sobre la respuesta específica del electrodo [12].

\subsection{Procesamiento Digital de las Señales}

Las señales de voltaje y corriente se exploran con el paquete de programas MATLAB 7.0, utilizando las Herramientas de Procesamiento Digital de Señales (PDS) y se procesan mediante la implementación de un programa en el código de programación de MATLAB 7.0 para la detección automática de la frecuencia de cortocircuito, la duración de los mismos y la conductividad durante el reencendido, parámetros que caracterizan el comportamiento de la transferencia metálica y de carga eléctrica. Para la detección de la duración de cada cortocircuito 
se requiere el análisis del histograma de frecuencias del voltaje de soldadura, para localizar el valor límite por debajo del cuál se va a considerar que existe un cortocircuito. En este caso se determina gráficamente, que el voltaje umbral puede ser tomado como $\pm 5 \mathrm{~V}$, por lo que el algoritmo debe determinar el tiempo en que la señal de voltaje permanece por debajo de este valor para cada cortocircuito. Además, debe anotar el momento en que ocurre la entrada para el cálculo posterior de la frecuencia de cortocircuito, una vez calculado el valor promedio del período de ocurrencia del corto. En el caso de la conductividad del reencendido se detecta el pico y se calcula la conductividad eléctrica promedio, a partir del voltaje y la corriente de soldadura en este intervalo de tiempo.

\subsection{Análisis estadístico}

Los parámetros tenidos en cuenta fundamentalmente para la soldadura con corriente alterna [1], se dividen en dos grupos (Tabla 2): los que se utilizan para caracterizar la transferencia metálica; y los que se utilizan para caracterizar la transferencia de carga.

Tabla 2. Parámetros de estudio.

\begin{tabular}{c|c|c}
\hline Parámetro & Transferencia Metálica & Transferencia de Carga \\
\hline Duración del Cortocircuito & $\mathrm{X}$ & - \\
\hline Frecuencia de cortocircuito & $\mathrm{X}$ & - \\
\hline Conductividad durante el reencendido & - & $\mathrm{X}$ \\
\hline
\end{tabular}

La compleja naturaleza de los fenómenos involucrados en la soldadura se manifiesta en cada parámetro, a través de distribuciones probabilísticas que difieren considerablemente del modelo de una distribución normal, lo cual ha sido comprobado en cada caso mediante el análisis del grado de abultamiento y la asimetría, unido al método de Shapiro-Wilk [18]. Tanto la no normalidad en la distribución de los puntajes de las muestras como la diferencia estadísticamente significativa entre las varianzas entre grupos invalidan los métodos del ANOVA clásico para realizar el análisis dentro y entre grupos [17].

El análisis de los modelos más parecidos a la distribución empírica en cada caso para, el parámetro "duración del cortocircuito",según la prueba Kolwomorov-Smirnov, permite utilizar un modelo Log-Normal [10, 12]. No fue posible encontrar similitud estadística entre las distribuciones continuas fundamentales y las distribuciones empíricas en el caso de los otros dos parámetros.

El empleo de un análisis libre de distribución, usando métodos no-paramétricos en la caracterización de las distribuciones empíricas, permite hacer un análisis robusto dentro de grupos mediante la prueba de Kruskal-Wallis [19, 20], partiendo de probar la hipótesis nula de que las medianas de cada distribución son estadísticamente iguales.

Mediante la prueba de la mediana de Mood [21], se puede probar la hipótesis de que las medianas de las 9 distribuciones son estadísticamente iguales, obteniéndose el valor de la mediana y su intervalo de confianza con una certidumbre del $95 \%$, válido para realizar el análisis entre grupos. Para obtener una medida de dispersión robusta se emplea el estimador MAD (Median Absolute Deviation) [22, 23], (Ecuación 1), que considera la mediana de los valores absolutos de la desviación de los datos respecto a su mediana.

$$
\mathrm{MAD}=\text { mediana }(\operatorname{abs}(\mathrm{X} \text {-mediana }(\mathrm{X}))
$$

\subsubsection{Duración del cortocircuito}

El resultado de la prueba de medianas de Mood (Tabla 3), permite probar la hipótesis de que las medianas de las 9 distribuciones es la misma, mediante el conteo del número de observaciones en cada muestra hacia cualquier lado de la "grand median", que en este caso es 26,0. Como el P-value para la prueba de Chi-cuadrado es menor que 0,05 , las medianas de las muestras son significativamente diferentes con un nivel de confianza de un $95 \%$, además incluye los intervalos de confianza de cada mediana basado en la estadística de orden de cada muestra.

Tabla 3. Resultados de la Prueba de las medianas de Mood. Total n =1844, Grand median =26,0 para la duración del cortocircuito.

\begin{tabular}{c|c|c|c|c|c|c|c}
\hline Muestra & $\begin{array}{c}\text { Tamaño } \\
\text { muestra }\end{array}$ & $\mathrm{n} \leq$ & $\mathrm{n}>$ & $\begin{array}{c}\text { Mediana } \\
\text { (conteo })\end{array}$ & $\begin{array}{c}95,0 \text { \% Limite } \\
\text { Inferior }\end{array}$ & $\begin{array}{c}95,0 \text { \% Límite } \\
\text { Superior }\end{array}$ & Mediana (ms) \\
\hline 12518 & 109 & 34 & 75 & 37,0 & 29,76 & 41,24 & 7,4 \\
\hline 12519 & 105 & 26 & 79 & 39,0 & 33,0 & 50,14 & 7,8 \\
\hline 12520 & 112 & 38 & 74 & 36,0 & 31,13 & 41,87 & 7,2 \\
\hline 14014 & 250 & 122 & 128 & 27,0 & 25,0 & 31,0 & 5,4 \\
\hline 14015 & 236 & 113 & 123 & 27,5 & 23,0 & 32,0 & 5,5 \\
\hline 14016 & 242 & 123 & 119 & 26,0 & 24,0 & 30,0 & 5,2 \\
\hline 16022 & 279 & 172 & 107 & 23,0 & 22,0 & 25,0 & 4,6 \\
\hline 16023 & 259 & 163 & 96 & 22,0 & 20,0 & 25,0 & 4,4 \\
\hline 16033 & 252 & 148 & 104 & 23,5 & 21,0 & 26,0 & 4,7 \\
\hline
\end{tabular}

Test statistic $=93,9427 \quad$ P-Value $=0,0$ 
La prueba de Kruskal-Wallis ha sido utilizada para hacer estudios estadísticos dentro de grupos, determinando si las medianas de las muestras tomadas con el mismo valor de corriente son iguales (Tablas 4 y 5). Los datos de las tres columnas son combinados y organizados de menor a mayor. Se calcula el rango promedio de cada distribución. Como el P-value es mayor o igual que 0,05 , puede afirmarse que no existen diferencias significativas entre las medianas de ninguno de los tres grupos muestrales.

Tabla 4. Resultados del análisis dentro de grupos, según la prueba de Kruskal-Wallis (duración del cortocircuito).

\begin{tabular}{|c|c|c|c|c|c|}
\hline Corriente & Experimento & Tamaño muestra & Rango Promedio & Test statistic & P-Value \\
\hline \multirow{3}{*}{$125 \mathrm{~A}$} & 12518 & 109 & 158,94 & \multirow{3}{*}{1,36543} & \multirow{3}{*}{0,505242} \\
\hline & 12519 & 105 & 172,34 & & \\
\hline & 12520 & 112 & 159,65 & & \\
\hline \multirow{3}{*}{$140 \mathrm{~A}$} & 14014 & 250 & 367,48 & \multirow{3}{*}{0,300368} & \multirow{3}{*}{0,86055} \\
\hline & 14015 & 236 & 367,55 & & \\
\hline & & 242 & 358,45 & & \\
\hline \multirow{3}{*}{$160 \mathrm{~A}$} & 16022 & 279 & 405,13 & \multirow{3}{*}{1,06014} & \multirow{3}{*}{0,588564} \\
\hline & 16023 & 259 & 384,87 & & \\
\hline & 16033 & 252 & 395,76 & & \\
\hline
\end{tabular}

Tabla 5. Resultado del análisis de dispersión de los datos.

\begin{tabular}{c|c|c|c|c|c|c|c|c|c}
\hline Muestras & 12518 & 12519 & 12520 & 14014 & 14015 & 14016 & 16022 & 16023 & 16033 \\
\hline MAD & 18 & 20 & 18 & 11 & 12,5 & 10 & 7 & 7 & 7,5 \\
\hline MAD(ms) & 3,6 & 4 & 3,6 & 2,2 & 2,5 & 2 & 1,4 & 1,4 & 1,5 \\
\hline
\end{tabular}

Una manera de sustentar el análisis, que permite llegar a conclusiones cualitativas importantes, lo constituye la exploración de las distribuciones de densidad empíricas de las 9 muestras (Figura 1).

\subsubsection{Frecuencia de Cortocircuito}

Para este parámetro se sigue el mismo procedimiento noparamétrico. Primeramente se aplica la prueba de las medianas

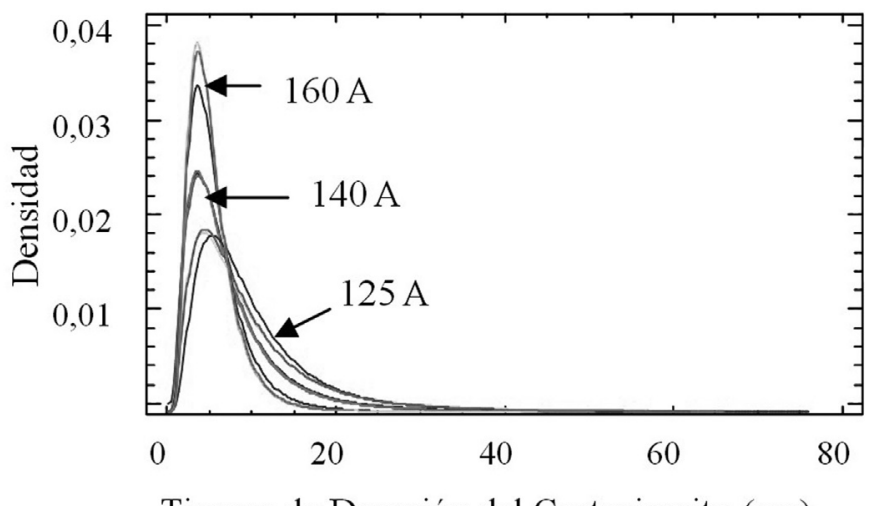

Tiempo de Duración del Cortocircuito (ms)

Figura 1. Distribución de densidad de las 9 muestras en los tres distintos niveles de corriente para el parámetro "duración del cortocircuito"[10].

\subsubsection{Conductividad durante el reencendido del arco}

Manteniendo los mismos criterios para la prueba entre grupos, que en los parámetros anteriores, la prueba de Mood de Mood, que arroja una diferencia significativa entre las medianas de las distribuciones.

Para realizar el análisis dentro de los grupos se utiliza la prueba de Kruskal-Wallis que no detecta diferencias significativas entre las medianas de las distribuciones obtenidas en cada condición de corriente.

La distribución de densidad (Figura 2), permite corroborar cualitativamente los resultados mostrados en las Tablas 6, 7 y 8.

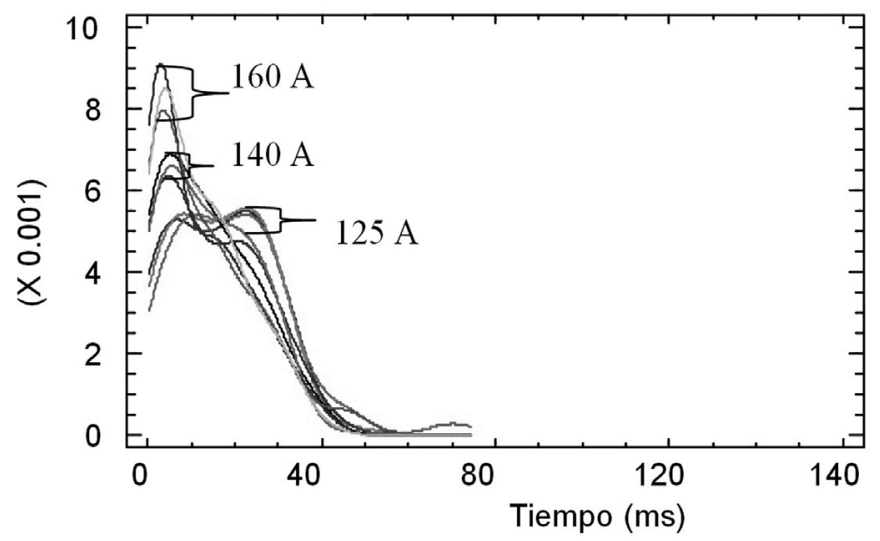

Figura 2. Funciones de distribución de densidad de las distribuciones obtenidas en los 9 experimentos para el parámetro "período de cortocircuito".

arroja que existen diferencias significativas en las medianas de las distribuciones empíricas de la conductividad durante el reencendido del arco (Tabla 9). 
Tabla 6. Resultados de la prueba de las medianas de Mood, Total $n=1824$, Grand median $=59,7$ para las 9 distribuciones del parámetro "frecuencia de cortocircuito".

\begin{tabular}{c|c|c|c|c|c|c|c}
\hline Muestra & $\begin{array}{c}\text { Tamaño } \\
\text { muestra }\end{array}$ & $n \leq$ & $n>$ & $\begin{array}{c}\text { Mediana } \\
(\text { ms })\end{array}$ & $\begin{array}{c}\text { 95,0\% Limite } \\
\text { Inferior(ms) }\end{array}$ & $\begin{array}{c}\text { 95,0\% Limite } \\
\text { superior(ms) }\end{array}$ & Frecuencia $(\mathrm{Hz})$ \\
\hline 12518 & 108 & 44 & 64 & 88,8 & 57,30 & 110,76 & 11,26 \\
\hline 12519 & 104 & 38 & 66 & 85,7 & 64,4 & 114,93 & 11,67 \\
\hline 12520 & 111 & 43 & 68 & 88,2 & 61,86 & 112,14 & 11,34 \\
\hline 14014 & 249 & 124 & 125 & 60,2 & 49,24 & 67,0 & 16,61 \\
\hline 14015 & 235 & 116 & 119 & 61,4 & 47,17 & 82,80 & 16,29 \\
\hline 14016 & 241 & 119 & 122 & 59,8 & 50,6 & 76,2 & 16,72 \\
\hline 16022 & 278 & 155 & 123 & 45,9 & 33,4 & 60,2 & 21,79 \\
\hline 16023 & 258 & 142 & 116 & 52,4 & 34,4 & 62,04 & 19,08 \\
\hline 16033 & 240 & 131 & 109 & 51,7 & 38,38 & 63,94 & 19,34 \\
\hline
\end{tabular}

Test statistic $=25,2727 \quad$ P-Value $=0,00139743$

Tabla 7. Resultados del análisis dentro de grupos, según la prueba de Kruskal-Wallis para el parámetro "frecuencia de cortocircuito".

\begin{tabular}{|c|c|c|c|c|c|}
\hline Corriente & Experimento & Tamaño muestra & Rango Promedio & Test statistic & P-Value \\
\hline \multirow{3}{*}{$125 \mathrm{~A}$} & \multirow{3}{*}{$\begin{array}{l}12518 \\
12519 \\
12520\end{array}$} & 108 & 158,37 & \multirow{3}{*}{0,246176} & \multirow{3}{*}{0,884186} \\
\hline & & 104 & 164,04 & & \\
\hline & & 111 & 163,62 & & \\
\hline \multirow{3}{*}{$140 \mathrm{~A}$} & \multirow{3}{*}{$\begin{array}{l}14014 \\
14015 \\
14016\end{array}$} & 249 & 355,06 & \multirow{3}{*}{0,549869} & \multirow{3}{*}{0,759622} \\
\hline & & 235 & 367,86 & & \\
\hline & & 241 & 366,46 & & \\
\hline \multirow{3}{*}{$160 \mathrm{~A}$} & \multirow{3}{*}{$\begin{array}{l}16022 \\
16023 \\
16033\end{array}$} & 278 & 385,29 & \multirow{3}{*}{0,653211} & \multirow{3}{*}{0,721368} \\
\hline & & 258 & 400,23 & & \\
\hline & & 251 & 397,25 & & \\
\hline
\end{tabular}

Tabla 8. Valores del MAD para las 9 distribuciones del parámetro "Período de cortocircuito".

\begin{tabular}{l|l|l|l|l|l|l|l|l|l}
\hline Muestras & 12518 & 12519 & 12520 & 14014 & 14015 & 14016 & 16022 & 16023 & 16033 \\
\hline MAD (ms) & 48,8 & 51,4 & 47,6 & 46,8 & 43,4 & 46,6 & 38,1 & 43,2 & 36,8 \\
\hline Promedios & \multicolumn{3}{|c|}{49,26} & \multicolumn{3}{c}{45,6} \\
\hline
\end{tabular}

Tabla 9. Resultados de la prueba de las medianas de Mood,Total n = 2508; Grand median = 1354,2.

\begin{tabular}{c|c|c|c|c|c|c}
\hline Muestra & $\begin{array}{c}\text { Tamaño de la } \\
\text { muestra }\end{array}$ & $n \leq$ & $n>$ & $\begin{array}{c}\text { Mediana } \\
\left({\left.\text { Siemen } * 10^{-3}\right)}^{-1}\right.\end{array}$ & $\begin{array}{c}\text { Limite inferior } \\
95,0 \%\end{array}$ & $\begin{array}{c}\text { Limite superior } \\
95,0 \%\end{array}$ \\
\hline 12518 & 247 & 169 & 78 & 1105,3 & 1050,06 & 1155,89 \\
\hline 12519 & 200 & 125 & 75 & 1165,1 & 1069,95 & 1292,84 \\
\hline 12520 & 248 & 165 & 83 & 1094,0 & 1031,82 & 1188,8 \\
\hline 14014 & 366 & 216 & 150 & 1265,5 & 1189,88 & 1306,31 \\
\hline 14015 & 389 & 208 & 181 & 1300,0 & 1223,39 & 1389,92 \\
\hline 14016 & 332 & 165 & 167 & 1355,5 & 1277,73 & 1465,89 \\
\hline 16022 & 237 & 61 & 176 & 1836,7 & 1646,23 & 2100,01 \\
\hline 16023 & 233 & 77 & 156 & 1593,6 & 1500,02 & 1816,75 \\
\hline 16033 & 256 & 68 & 188 & 1868,2 & 1664,74 & 2185,57 \\
\hline
\end{tabular}

Test statistic $=225,764$ P-Value $=0,0$

El análisis entre grupos se realiza mediante la prueba de Kruskal-Wallis, no detectándose diferencias significativas entre las medianas de las distribuciones de conductividad durante el reencendido del arco, obtenidas en cada uno de los tres diferentes regímenes de corriente (Tabla 10). 
Tabla 10. Resultados del análisis dentro de grupos según la prueba de Kruskal-Wallis.

\begin{tabular}{|c|c|c|c|c|c|}
\hline Corriente & Experimento & Tamaño muestra & Rango Promedio & Test statistic & P-Value \\
\hline \multirow{3}{*}{$125 \mathrm{~A}$} & 12518 & 247 & 336,86 & \multirow{3}{*}{2,69549} & \multirow{3}{*}{0,259825} \\
\hline & 12519 & 200 & 367,15 & & \\
\hline & 12520 & 248 & 343,65 & & \\
\hline \multirow{3}{*}{$140 \mathrm{~A}$} & 14014 & 366 & 516,23 & \multirow{3}{*}{5,48374} & \multirow{3}{*}{0,0644497} \\
\hline & 14015 & 389 & 546,23 & & \\
\hline & 14016 & 332 & 571,85 & & \\
\hline \multirow{3}{*}{$160 \mathrm{~A}$} & 16022 & 237 & 375,00 & \multirow{3}{*}{2,9751} & \multirow{3}{*}{0,225925} \\
\hline & 16023 & 233 & 344,17 & & \\
\hline & 16033 & 256 & 370,40 & & \\
\hline
\end{tabular}

El análisis de dispersión de los datos se realiza de la misma manera, a partir del estimador MAD.

Del análisis de las distribuciones de densidad para este parámetro (Figura 3), se observa una definición menos evidente de los regímenes de trabajo. Atendiendo al solapamiento de las distribuciones del régimen de 125 y $140 \mathrm{~A}$, existe más definición en el régimen de 160 A, aunque tienen también mayor dispersión, como puede constatarse en los datos ofrecidos en la Tabla 11.

Tabla 11. Valores del MAD para el parámetro "conductividad durante el reencendido del arco".

\begin{tabular}{l|l|l|l|l|l|l|l|l|l}
\hline Muestras & 12518 & 12519 & 12520 & 14014 & 14015 & 14016 & 16022 & 16023 & 16033 \\
\hline MAD. $10^{-3}\left(\mathrm{~S}^{-1}\right)^{*}$ & 289,41 & 341,12 & 281,445 & 275,735 & 273,6 & 386,2 & 683,5 & 515 & 812,4 \\
\hline
\end{tabular}

* Siemen/seg

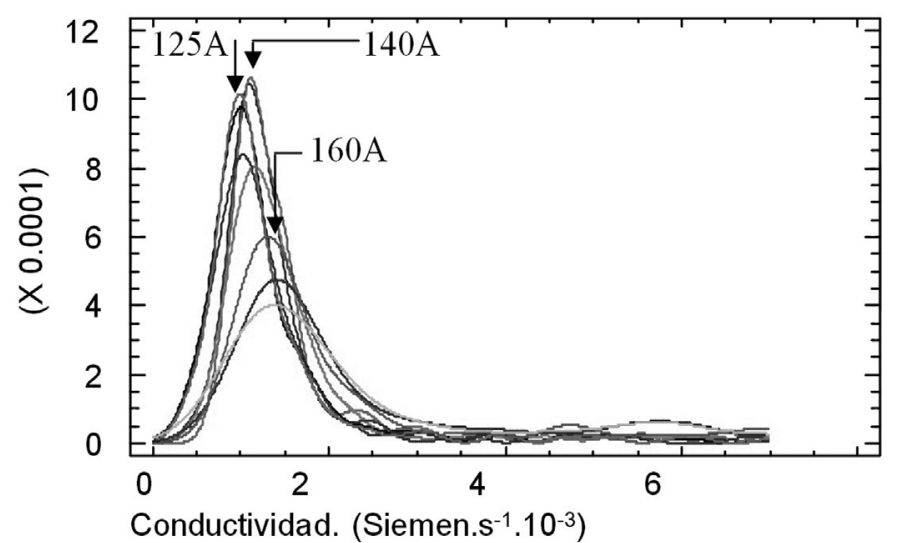

Figura 3. Funciones de distribución de densidad del parámetro "conductividad durante el reencendido"de los conjuntos obtenidos en los 9 experimentos.

\section{Análisis de resultados}

Por las características de las distribuciones estudiadas, es necesario hacer un análisis no parámetrico evitando errores en el ajuste a curvas de modelos conocidos y la influencia de valores atípicos del parámetro, que puedan afectar la verdadera tendencia de la distribución.

Como es conocido [11, 14], el cortocircuito ocurre cuando la mezcla de revestimiento y metal fundidos de la punta del electrodo, pendiente en forma de gota líquida, toca el baño fundido minimizando la longitud de arco y el voltaje en los terminales de la fuente de soldadura, mientras la corriente de soldadura y el campo magnético asociado a su paso por el electrodo, facilitan el proceso de desprendimiento de la gota. El tiempo de duración del cortocircuito disminuye con el aumento de la magnitud de la corriente (Tabla 3), de acuerdo con lo reportado en la literatura $[5,4,3,11]$. La dispersión disminuye con el aumento de la corriente, lo cual puede interpretarse como una mayor estabilidad de la duración del cortocircuito (Tabla 5). La frecuencia de los cortocircuitos, aumenta con el aumento de la corriente de soldadura (Tabla 6), resultado consistente con el hecho de que disminuya el tiempo de duración del cortocircuito (Tabla 3).

Durante la inversión de la polaridad de la corriente alterna, el arco se enfría y se apaga ocasionalmente, necesitando reencenderse en el subsiguiente cambio de polaridad, disminuyendo considerablemente la conductividad del arco y produciéndose un pico de voltaje llamado pico de reencendido [11]. La conductividad durante el reencendido del arco es un parámetro que involucra el comportamiento del voltaje y la corriente de soldadura y es directamente proporcional a la corriente de soldadura e inversamente proporcional al voltaje. Valores mayores de este parámetro sugieren un arco más conductivo y, por tanto, más fácil de reencender, lo cual ayuda a la estabilidad del mismo. Se observa un aumento de la conductividad a medida que aumenta la corriente de soldadura (Tabla 9); sin embargo, la dispersión de este parámetro es mayor en el régimen de $160 \mathrm{~A}$ (Tabla 11), lo cual coincide con el aumento de la frecuencia de cortocircuito, que propicia inestabilidad en el reencendido.

Del análisis del comportamiento de la dispersión de la duración del cortocircuito (Tabla 5), se aprecia una mayor estabilidad (1,43 $\mathrm{ms})$ en el régimen de $160 \mathrm{~A}$, al igual que en la dispersión (39,36 ms) del período de cortocircuito (Tabla 8); también se aprecia que los mayores valores de conductividad $\left(1766,17.10^{-3} S \cdot S-1\right)$ 
ocurren en este régimen (Tabla 9). Estos criterios evidencian una mayor estabilidad en el régimen de $160 \mathrm{~A}$.

El análisis dentro de grupos, aplicando el método de Kruskal-Wallis, para los tres parámetros, no aportó diferencias significativas entre las medianas de los parámetros obtenidos en cada nivel de corriente (Tablas 4, 7, 10), lo cual es coherente con el análisis gráfico de las distribuciones empíricas de densidad (Figuras 1, 2, 3) y las dispersiones (Tablas 5, 8, 11). Este resultado permite dar un criterio positivo sobre la repetitividad de los resultados.

El análisis entre grupos, basado en el análisis del comportamiento de las distribuciones de densidad empírica en los distintos regímenes de trabajo y las estimaciones de las medianas y su desviación absoluta respecto a la misma, permite observar una adecuada reproducibilidad de los resultados, ya que las distribuciones de densidad mantienen variaciones proporcionales características en los diferentes regímenes de trabajo.

El comportamiento de los parámetros duración del cortocircuito y frecuencia del cortocircuito están relacionados al disminuir la mediana de la duración del cortocircuito con el aumento del régimen de corriente (Tabla 3) y, a su vez, la dispersión absoluta de los datos respecto a su mediana disminuye (Tabla 5), mientras que la frecuencia de cortocircuito aumenta (Tabla 6) y su dispersión disminuye (Tabla 8). A medida que la corriente aumenta, disminuye el tiempo en que la gota está en contacto con el metal fundido pues las condiciones para su desprendimiento mejoran; consecuentemente, aumenta la frecuencia con que ocurre la transferencia. Menores dispersiones indican mayor estabilidad en el régimen de 160 A, lo que corrobora los resultados obtenidos mediante análisis metalográfico [12].

Durante la realización de los experimentos se observó un mejor desempeño eléctrico operativo en el régimen de 160 A, apreciándose mayor facilidad en los procesos de cebado y mantenimiento del arco. Los valores de frecuencia de cortocircuitos promedio coinciden con valores reportados, a partir de mediciones hechas por técnicas ópticas [11], durante el desempeño del proceso SMAW a 160 A con electrodos de $4 \mathrm{~mm}$. Los índices de estabilidad reportados por algunos investigadores $[1,3,4]$, según ha sido expuesto [10], utilizan el coeficiente de variación como índice de estabilidad en la transferencia metálica y de carga, se ha demostrado que el empleo de estos índices es solo válido en determinadas relaciones de los valores medios y su desviación estándar. El empleo de la media, como medida de localización, y la desviación estándar, como medida de dispersión, indican el empleo de técnicas paramétricas para la descripción estadística de las distribuciones obtenidas $[1,3,4]$, sin embargo, no aparece descrito el tipo de modelo paramétrico usado para hacer los cálculos.

El espectro de frecuencias del arco se ubica fundamentalmente por debajo de $240 \mathrm{~Hz}$ en la soldadura SMAW, según demuestran estudios espectrales mediante la aplicación de la transformada de Fourier a las señales de voltaje y corriente en los experimentos realizados en este estudio. Esto se debe a la inercia del arco eléctrico [11]. Coincidiendo con la literatura [8], el empleo de una frecuencia de 5000 muestras por segundos garantiza una adecuada medición de los procesos transientes en todas las posibles alteraciones del voltaje y la corriente de soldadura, tanto en amplitud, como en fase y frecuencia.

\section{Conclusiones}

1. Teniendo en cuenta el significado físico de los parámetros evaluados, en términos estadísticos, existe una adecuada repetitividad y reproducibilidad en los resultados obtenidos del análisis dentro y entre grupos, apreciándose un comportamiento coherente con las condiciones fenomenológicas del proceso.

2. El análisis no paramétrico realizado, permite un procesamiento robusto de los datos, evitando la influencia de valores atípicos y errores derivados del empleo de aproximaciones a distribuciones continuas conocidas.

3. El electrodo E6013 Ø 4mm, en el régimen de 160 A, presenta una mayor estabilidad, manifiesta en la regularidad del aporte metálico, dada por el MAD promedio del período de cortocircuito (39,36 ms) y de la duración del cortocircuito (1,43 $\mathrm{ms})$, reafirmada con la presencia de una mayor conductividad eléctrica durante el reencendido $\left(1766,17 \mathrm{~S} \cdot \mathrm{s}^{-1}\right)$.

\section{Referencias Bibliográficas}

[1] Farias, J. P. Metallic Mg as a coating component in C-MnNi electrode. 1993. p 225. Tese (Doutorado). Universidade Federal de Santa Catarina, Brasil.

[2] Rissone, N. M. R. de, Surian, E. S., Vedia, L. A. Effect of slag variations on ANSI/AWS A5.1-91 E6013 electrode properties: replacement of $\mathrm{TiO} 2$ in electrode coating with $\mathrm{MnO}$, $\mathrm{FeO}, \mathrm{CaO}, \mathrm{MgO}, \mathrm{K}_{2} \mathrm{O}, \mathrm{Na}_{2} \mathrm{O}$. Science and Technologic of Welding and Joining, 2001. 6(5).

[3] Rissone, N. M. R. de et al. ANSI/AWS A5.1-91 E6013 Rutile electrodes: The Effect of Calcite. Welding Journal Supplement. p 113-s - 124-s. Julio 2002.

[4] Souza, M. S. et al. Validación de un sistema computarizado para el control de la calidad de electrodos revestidos, 1995. p 87. Tese (Doutorado). Centro de Ciências Exatas e Tecnología. Universidade Federal de Uberlândia, Brasil..

[5] Fedele, R. A., Brandi, S. D. Influência dos parâmetros de soldagem por eletrodo revestido na transferêcia de elementos químicos. Soldagem \& Inspeção, 2002. 7(2).

[6] Pessoa, E. C. P. Estudo comparativo do Desempenho de Eletrodos Revestidos E6013, E7024 e E7018 em Soldagem Subaquática em Água Doce do Aço A36 à Profundidades de 50 e 100 metros. in Congresso Brasileiro do Engenharia do Fabricação. 2003. Uberlandia. Brasil.

[7] Farias, J. P., Bálsamo, P. S., Surian, E. S. The effect of wollastonite on operational characteristics of AWS E6013 electrodes. Journal of the Brazilian Society of Mechanical Sciences and Engineering, 2004. 26(3): p. 17.

[8] Ponomarev, V., Arc Welding Process Statistical Analysis. Methodical Approaches, Analysis Conceptions, Experiences. 1997.

[9] ANSI/AWS, Specification for carbon steel electrodes for shielded metal arc welding, Standard 5.1-91,1991, American National Standards Institute/American Welding Society, Miami, 
Fl.

[10] García Rodríguez, A. G. P. Criterios sobre algunas Metodologías de Caracterización Operativa de Electrodos Revestidos en la Soldadura en Modo de Transferencia Metálica por Cortocircuitos. Soldadura \& Inspeçao,Abril-Junio 2008.13(2).pag 141-149.

[11] Lancaster, J. F. The Physics of Welding. 2 Ed, 1986. 340

p..

[12] García Rodríguez, A. G. P. Valoración del Desempeño de un Dispositivo de Autoalimentación Diseñado para la Evaluación Operativa de Electrodos Revestidos. Soldagem \& Inspeção, Aceptado para publicar 2009.

[13] Cary, H. B. Modern Welding Technology third edition. 1996.

[14] Weman, K. Welding Processes Handbook. 2003, p 189. Woodhead Publishing Ltd Cambridge.

[15] Pessoa, C. P. E. Study of Porosity Location in Fresh Water Wet Welds. in 22ND International Conference on Offshore Mechanics and Arctic Engineering. 2003. Cancun, México.

[16] Roca, A. S. Nuevo criterio para el análisis de estabilidad del proceso de soldadura GMAW, empleando técnicas de Emisión Acústica e Inteligencia Artificial. 2006. p.105. Tese (Doctorado). Facultad Ingeniería Mecánica, Departamento de Tecnología de Construcción de Maquinaria. Universidad de Oriente, Santiago de Cuba.

[17] Guerra, C. W., Barreras, E. M. Estadística. 1ra ed. 2003, p 376. Habana: Félix Varela.

[18] Shapiro, S. S. W. An analysis of variance test for normality (complete samples). Biometrika, 1965. 52 (3 and 4): p. 591-611.

[19] Wallis, W. H. K. Use of ranks in one-criterion variance analysis. Journal of the American Statistical Association, 47 (260) 1952 p. 583-621..

[20] Siegel, S., Castellan, N. J. Nonparametric Statistics for the Behavioral Sciences. 1988, New York: McGraw-Hill.

[21] Mood, A. M. Introducction to the Theory of Statistics. Third Edition ed. 1974: McGraw-Hill. 540-541.

[22] Ripley, B. D. Robust Statistics. 2004, Springer-Verlang. p. 11.

[23] Sachs, L. Applied Statistics: A Handbook of Techniques, ed. Springer-Verlag. 1984. 253. 